\title{
O "materialismo cultural" de Raymond Williams a partir da história intelectual: caminhos e possibilidades
}

\author{
Raymond Williams' Cultural Materialism from an Intellectual \\ History perspective: approaches and possibilities
}

https://doi.org/10.34112/2317-0972a2019v37n77p33-46

\section{Carla Baute ${ }^{1}$}

RESUMO: Esse artigo discutirá alguns aspectos de uma pesquisa de mestrado em andamento. Trata-se de um trabalho que privilegia o estudo da teoria cultural do pensador galês Raymond Williams, o Materialismo Cultural, e o modo como ele se desenvolveu em importantes obras teóricas de diferentes momentos de sua trajetória intelectual. Busca-se no texto dialogar com o contexto de produção dessa teoria na segunda metade do século XX. A relevância dessa empreitada reside na tentativa de inter-relacionar um rigoroso estudo teórico com uma análise do contexto histórico, utilizando metodologia de caráter interdisciplinar - como é de praxe na área da história intelectual - advinda da Sociologia da Cultura e da Teoria Cultural, respectivamente o uso do conceito de campo de Pierre Bourdieu e de estrutura de sentimento, do próprio Williams. No que diz respeito à contribuição específica advinda da prática historiográfica, ressalta-se a ênfase na relação dialética entre obra e vida, sem privilegiar somente a obra - vendo-a somente como um reflexo do contexto -, nem exclusivamente o contexto, entendido como simples plano de fundo.

Palavras-chave: História Intelectual; Materialismo Cultural; Raymond Williams; Teoria da História.

1. Universidade Estadual de Campinas, Campinas, SP, Brasil. 
ABSTRACT: This paper will discuss a few aspects of an ongoing master's research, a study regarding the cultural theory of welsh thinker Raymond Williams: Cultural Materialism. Mostly focused on how it was developed in the most important theoretical works by the author in different moments of his intellectual career, aiming to establish a dialogue with its respective contexts within the second half of the twentieth century. The relevance of such enterprise resides in the attempt to relate a rigorous theoretical study with an analysis of the historical context, using an interdisciplinary methodology - a usual practice in the area of Intellectual History - from both Sociology of Culture and Cultural Theory, the use of the concept of Field, by Pierre Bourdieu, and Structure of Feeling, by Williams himself. As to the how the contribution of the historiographical practice is concerned, the emphasis is in the dialectical relationship between work and life, without privileging only the work - seeing it as a mere reflection of the context -, or in the context alone, understood as mere background.

KEYWORDS: Intellectual History; Cultural Materialism; Raymond Williams; Theory of History.

Esse artigo tratará de alguns aspectos de minha atual pesquisa de mestrado em História, realizada no IFCH da Unicamp, sob orientação da professora Dra. Silvana Rubino e financiada pela Fapesp², o que possibilitou a realização de um estágio de pesquisa na Swansea University, supervisionado pelo professor Daniel Williams.

Raymond Henry Williams (1921-1988) foi um pensador galês frequentemente vinculado à New Left britânica ${ }^{3}$ e considerado um dos precursores dos Estudos Culturais $^{4}$ na Inglaterra. Formado em literatura inglesa pelo Trinity College em Cambridge, teve seus estudos universitários interrompidos pela Segunda Guerra Mundial. Retomando-os após o término do conflito, recebeu seu diploma em 1946. Durante os anos seguintes se dedicou ao ensino de adultos por meio da organização

2. Processo n. 2017/08999-8.

3. No contexto do pós-guerra, mais especificamente entre as décadas de 1960/70, a New Left britânica surgiu como um grupo heterogêneo - dentre eles comunistas dissidentes, socialistas independentes e intelectuais radicais - que, diante dos avanços do imperialismo e das revelações dos abusos autoritários da URSS, realizou esforços visando suprir a necessidade de se pensar novos rumos políticos dentro da esquerda (Cf. CEVASCO, 2001, p. 123-125).

4. Grupo de estudos organizado, de início, pelo Center for Contemporary Cultural Studies, fundado em 1964 por Richard Hoggart (1918-2014) e parte do Departamento de Inglês da Universidade de Birmingham. Seu eixo principal era fomentar pesquisas em torno da relação entre cultura e sociedade, tendo como base três textos fundadores: The Uses of Literacy, lançado em 1957 e de autoria do próprio Hoggart; Culture and Society de Raymond Williams, de 1958 e The making of the english working-class, escrito por E. P. Thompson, de 1963 (Cf. ESCOSTEGUY, 1998, p. 87-88). 
Workers Education Association (WEA), regressando a Cambridge como professor universitário no ano de 1961. Aposentou-se em 1983 e passou o resto de seus dias no interior da Inglaterra, onde faleceu em 1988 (WILLIAMS, 1979, p. 11-16).

Suas publicações foram numerosas, totalizando mais de trinta livros. Para além dos estudos literários, Williams também contribuiu com análises sobre cinema, teatro, televisão e teoria crítica cultural, além de ter escrito contos, romances e peças de teatro. Em meio aos profissionais do Departamento de Inglês de Cambridge do período, Williams era tido como um "sociólogo fora do lugar". Em seu trabalho buscou conciliar duas correntes de análise: a de um rigoroso estudo do texto e a da análise da sociedade (EAGLETON, 1988, p. 5-6).

A presente pesquisa é um estudo da teoria cultural do pensador galês, o Materialismo Cultural, e o modo como se desenvolveu em importantes obras teóricas de diferentes momentos de sua trajetória intelectual, e como dialogou com seus respectivos contextos na segunda metade do século XX. Com foco especial em Cultura e Sociedade (1958), no artigo "Base e Superestrutura na Teoria Cultural Marxista” (1973) e em Marxismo e Literatura (1977), intento uma análise dialética que enxerga, tanto no estudo do texto quanto do contexto, elementos para uma contribuição dentro do campo da história. Utilizo seus livros, bem como bibliografia sobre a New Left britânica e o pensamento marxista ocidental, sobre a crítica literária de Cambridge e obras sobre o Reino Unido no período, além de papéis pessoais do autor, denominados de Raymond Williams Papers no País de Gales.

Uma outra linha de análise a ser perseguida, em conjunto e concomitante aos itens acima descritos, diz respeito a uma atenção especial ao peso do processo histórico no corpo da crítica das obras escolhidas, buscando contribuir, assim, com estudos específicos de teoria da história.

Nas palavras do próprio autor, em artigo publicado na New Left Review em 1976, Materialismo Cultural designa:

Uma teoria da cultura como um processo produtivo (social e material) e de práticas específicas, de 'artes', como usos sociais dos meios materiais de produção (desde a linguagem como 'consciência prática' material até as tecnologias específicas da escrita e formas da escrita, através de sistemas de comunicação mecânicos e eletrônicos) (WILLIAM, 1976, p. 88-89). 
A denominação dessa teoria como marxista só apareceria em Marxismo e Literatura, lançado no ano seguinte - 1977. No entanto, Williams ressaltou que qualquer contribuição sua dos últimos trinta e cinco anos teria se desenvolvido numa relação complexa e direta com argumentos e ideias marxistas (WILLIAMS, 1977, p. 5). Essa declaração exemplifica um dos questionamentos que motivaram este trabalho: de modo geral, como o pensamento marxista se desenvolveu para buscar responder aos novos problemas e questões do século XX e, especificamente, como isso se deu no Reino Unido, lugar tradicionalmente avesso a teorização.

Dentro da minha área de atuação e tendo como objeto a vida e obra de Williams, creio que a História Intelectual fornece possibilidades para um estudo frutífero. Conforme enfatizou Jorge Meyers, a consolidação de uma disciplina - ou subdisciplina - é sempre temporária (MEYERS, 2016, p. 24), fato que parece ser ainda mais urgente nesse campo plural e interdisciplinar. Nesse sentido, uma definição precisa poderia indicar um contrassenso; no entanto, para certo esclarecimento provisório, destaco a seguinte passagem de Meyers:

a história intelectual consiste em uma exploração da produção douta realizada pelas elites letradas do passado, enfocada a partir de uma perspectiva que considera a própria condição de inteligibilidade histórica dessa produção como derivada de sua reinserção (por parte do pesquisador) em um contexto social e cultural - simbólico e material - historicamente específico que, na maioria dos casos, será o contemporâneo dessa produção (MEYERS, 2016, p. 24-25).

Para minha reinserção nesse contexto, a contribuição de Pierre Bourdieu é fundamental. Denominada de filosofia da ação, sua sociologia buscou analisar as relações e potencialidades entre os agentes e as estruturas das situações sociais.

É central aqui o conceito de campo. Pode-se pensar o campo como um sistema de relações e disputas. Um espaço delimitado que contém regras próprias de funcionamento, leis gerais que geram a construção de um habitus, isto é, um

sistema de disposições adquiridas pela aprendizagem implícita ou explícita que funciona como um sistema de esquemas geradores, é gerador de estratégias que podem ser objetivamente afins aos interesses objetivos de seus autores sem terem sido expressamente concebidas para este fim (BOURDIEU, 2006, p. 125). 
Esses "espaços estruturados de posições" podem ser dos mais diversos tipos seja campo filosófico, científico, político etc. -, e cada um deles tem propriedades específicas. E para que funcionem, devem estar em jogo objetos de disputas e pessoas e/ou instituições dispostas a jogá-los. Os que estão jogando contribuem para a reprodução do campo (BOURDIEU, 2006, p. 122).

No que diz respeito ao campo das produções culturais, Bourdieu sublinhou como são travados jogos pela disputa de capital simbólico. Este não reside simplesmente no sucesso comercial e econômico, mas em complexas relações que envolvem a mobilização de diversos campos distintos, submersas em relações de forças, interesses e estratégias que variam (BOURDIEU, 1996, p. 169-173).

Ao refletir especificamente sobre a criação intelectual, Bourdieu traçou suas especificidades:

É preciso perceber e considerar que a relação que um criador mantém com a sua obra e, por isso mesmo, a própria obra são afetadas pelo sistema de relações sociais nas quais se realiza a criação como ato de comunicação ou, mais precisamente, pela posição do criador na estrutura do campo intelectual (BOURDIEU, 1968, p. 105).

O sociólogo francês aplicou tais premissas teóricas na análise de $A$ educação Sentimental, de Gustave Flaubert. Identificando o romancista do séc. XIX como um interlocutor privilegiado de seu momento histórico, criador de "uma espécie de experimentação sociológica” (BOURDIEU, 2002, p. 25), Bourdieu relacionou seus personagens com diferentes polos de poder do período, mostrando como suas ações dentro da trama se relacionavam com as possibilidades e os limites da sociedade parisiense pós-revolução de 1848 (BOURDIEU, 2002, p. 18-23).

No entrelaçamento de componentes psíquicos e sociais, a cidade de Frédéric Moreau, protagonista da história, também era a mesma de Flaubert. E justamente ao "dessacralizar" o "gênio criador" na análise sociológica de uma obra sua experiência literária pode ser intensificada, e não diminuída, como defenderam alguns estudiosos da arte (BOURDIEU, 2002, p. 12-4). Pois, como escreveu Bourdieu:

É através da elaboração de uma história e graças a ela, que o autor é levado a trazer à luz a estrutura mais profundamente enterrada, mais obscura, porque a mais diretamente ligada aos seus investimentos primários, que está no próprio princípio de suas estruturas mentais e de suas estratégias literárias (BOURDIEU, 2002, p. 40). 
Para o sociólogo brasileiro Enio Passiani e o crítico literário inglês Andrew Milner existe um "mal-entendido" nas apropriações da estrutura dentro do pensamento de Bourdieu. Estrutura seria sempre "estruturante" e não "estruturada", ou seja, passível de transformar-se e sempre em movimento com a história (PASSIANI, 2009, p. 294; MILNER, 2002, p. 167-169). Desse modo, não há uma supressão do indivíduo em relação à sociedade, mas uma relação dialética que supera essa falsa dicotomia.

No centro da teoria de Williams está posta a superação dessa dicotomia. Propondo uma leitura não ortodoxa da analogia base/superestrutura, cerne do pensamento marxista de cunho mais tradicional, Williams - assim como Bourdieu - buscou "descortinar" o véu fetichizante da cultura e mostrá-la em sua complexidade de relações com a materialidade da sociedade, oscilando entre condicionante e condicionada, comum e extraordinária.

Em Cultura e Sociedade, Williams traçou linhas de análise sofisticadas que buscaram não ceder à tentação de delinear relações diretas da sociedade com a arte - presentes em teorias do reflexo reducionistas -, mas procuraram mostrar sua fluidez, num movimento de influências mútuas. Um exemplo está na sua assertiva acerca de movimentos que enfatizaram a "internalização" do processo criativo, de concepções do artista como uma "pessoa especial" no contexto de ascensão do capitalismo industrial, dentro do qual o mesmo estava se transformando em mais um trabalhador vendendo a sua força de trabalho. Ao passo que a própria literatura se tornava um negócio, acentuaram-se as críticas ao comércio em geral. Ao mesmo tempo em que se ampliava o público leitor, criticou-se a noção de "gosto" do povo. Interagindo nessa mistura, estavam também transformações no conceito de cultura em conjunto com tentativas de posicioná-la como a antítese do comércio. Algo sublime versus algo mundano (WILLIAMS, 1963, p. 33-52). Segundo Williams:

Sob pressão, a arte tornou-se uma abstração simbólica de toda uma gama de experiências humanas gerais [...] na prática houve insights profundos e grandes obras de arte; mas, na pressão contínua da vida, a liberdade do gênio achou cada vez mais difícil harmonizar-se com a liberdade do mercado, e a dificuldade não foi resolvida, apenas amortecida por uma idealização (WILLIAMS, 1963, p. 51).

As semelhanças entre os dois pensadores são tão palpáveis que o próprio Bourdieu ressaltou a enorme contribuição de Williams para que seu trabalho fosse divulgado no mundo anglo-saxão (BOURDIEU, 2002, p. 13). Conjuntamente com 
Nicholas Garnham, Williams escreveu sobre as relações entre os Estudos Culturais e a sociologia de Bourdieu em um artigo publicado no ano de 1980. No texto, os autores lamentaram o fato da influência do pensamento do francês no mundo anglófono estar restrito à Antropologia e à Sociologia da Educação (GARNHAM; WILLIAMS, 1980, p. 209-210).

Nesse sentido, defendo a pertinência do uso tanto das ferramentas metodológicas de Bourdieu quanto de Williams destacadas acima para a proposta de pesquisa aqui apresentada, uma análise dialética da cultura. Pois tanto o conceito de campo, quanto o de estrutura de sentimento ${ }^{5}$ se relacionam, ao mesmo tempo em que oferecem contribuições distintas para um estudo dialético entre texto e contexto dentro da história intelectual.

A partir desses elementos é possível pensar na gama de possibilidades de fontes e bibliografia disponíveis para a realização da pesquisa. Em conjunto com seus livros publicados e a bibliografia acessível no Brasil, no arquivo Raymond Williams Papers no País de Gales estão disponíveis seus documentos pessoais, tais como suas correspondências e valiosos cadernos de anotações que datam dos anos 1950 até 1980. Lá também se encontra extensa coleção de todos os textos publicados por Williams, desde livros (que acompanham anotações e versões de alguns capítulos), até resenhas (escritas por ele ou por terceiros a respeito de seu trabalho), entrevistas e documentos profissionais, tais como alguns programas de disciplinas oferecidas por ele, palestras e eventos variados. Na Weston Library, em Oxford, é possível acessar documentação referente ao período em que ele foi professor de adultos em um departamento extramuros da Universidade, lá se encontram programas de aula e documentos administrativos.

Como mencionei, a pesquisa de mestrado ainda está em desenvolvimento. No atual estágio pude delimitar quais problemáticas, dentro do grande espectro que o recorte possibilita, são as mais frutíferas. No que diz respeito a Cultura e Sociedade, além da leitura aprofundada da obra em si, penso ser necessário olhar com atenção para a formação universitária de Williams, o ambiente intelectual, social e político

5. A expressão apareceu pela primeira vez em uma obra de Williams com Michael Orrom, Preface to Film. London: Film Drama, 1954. Cf., por exemplo: (WILLIAMS, 1961, p. 19-144; WILLIAMS, 1984, p. 128-135). Em Marxismo e Literatura o conceito aparece mais bem desenvolvido e explicado. Williams desenvolveu a noção de "estrutura de sentimento" na tentativa de "articular a experiência intelectual e sua prática concreta” (PASSIANI, 2009, p. 286). Dentro dessa perspectiva, destacou o lugar privilegiado da nova obra cultural e suas relações com as mudanças estruturais e simbólicas na sociedade. No entanto, enfatizou que esse potencial de ação não reside em um só indivíduo, mas sim em grupos e na interação desse com outros grupos (PASSIANI, 2009, p. 286; WILLIAMS, 1980, p. 148-169). 
que o levou à educação de adultos e seu desenvolvimento enquanto intelectual nesse período. Para isso, é indispensável o acesso a documentos tais como suas anotações e programas de disciplinas do período específico. Também é importante analisar os conteúdos do livro em que transparecem os conflitos que o autor teve em relação a sua formação e suas preocupações socialistas, tendo de um lado o elitismo de F. R. Leavis e seus discípulos e do outro o pensamento marxista no Reino Unido daquele período. Nesse sentido, o meu foco está, primordialmente, na parte III do livro, na seção 6 - "Marxismo e Cultura" e também na "Conclusão".

Assim, penso que alguns eixos de análise são importantes. $O$ primeiro diz respeito à relação de Williams com o "estado da arte" da crítica literária de Cambridge e as ambiguidades que Cultura e Sociedade carrega. Apesar de seu autor afirmar que o livro foi escrito em completo isolamento intelectual, é inegável a influência de F. R. Leavis e da revista Scrutiny (periódico britânico fundado em 1932). Uma dissertação toda poderia ser escrita a partir dessa problemática; no entanto, destacarei apenas alguns pontos, e me pautarei especialmente na bibliografia sobre o tema (resenhas e artigos críticos sobre o livro) e em alguns documentos selecionados do arquivo em Swansea. Um desses pontos é a própria noção de comunidade e cultura. Mesmo que Williams faça uma crítica à posição vinculada a Scrutiny de que uma minoria intelectualizada teria para si a "missão" de levar a "cultura" à população - e ainda por cima, de decidir o que é ou não cultura -, ele não abandona totalmente os pressupostos dessa "escola" ao teorizar sobre a cultura da classe trabalhadora e a ideia de cultura comum. Por trás dessas suas concepções está uma ideia de comunidade que em muito se parece com a dos "Leavisites", uma comunidade idealizada, quase uma entidade espiritual, encarnada não nas estruturas sociais, mas sim nas tradições (MULHERN, 1979, p. 310-311).

No que diz respeito a um dos objetos do livro, a tradição cultura e sociedade, Williams disse que pretendia analisar a complexidade das transformações em diferentes concepções de cultura e sua intrínseca relação com as mudanças da sociedade industrial britânica do período e defendia certas continuidades na tradição que datava de meados do século XVIII. Há no livro passagens que demonstram como o autor dominava com maestria a técnica do close reading da crítica literária de Cambridge. Sua atenção à minúcia do texto - seja ele literário ou comentário social - é louvável, e trouxe à tona aspectos interessantes que tornaram sua hipótese celebrada.

Em Criticism and Ideology, de 1976, Terry Eagleton escreveu uma crítica voraz de Williams e Cultura e Sociedade, em que destacava que o livro "foi na realidade um projeto idealista e academicista. Ele pode sustentar sua tese somente pela desatenção 
sistematizada ao caráter reacionário da tradição com a qual lida” (EAGLETON, 1976, p. 25). Penso que essa "desatenção sistematizada" deriva ainda mais do fato de que, apesar de Williams ter enfatizado que as mudanças na ideia de cultura foram não só respostas à industrialização, mas também aos novos desenvolvimentos político-sociais e à democracia (WILLIAMS, 1963, p. 7), a discussão ficou muito centrada nos textos em si e falhou em trazer elementos para análises políticas, econômicas, sociais e históricas, isso para não dizer de uma completa inexistência das mesmas.

Pode-se argumentar que a "razão" disso está em seu treinamento de crítico literário, pois mesmo que Williams criticasse abertamente o elitismo da Scrutiny, ele não foi capaz de romper totalmente com o caráter idealista da concepção de texto e autor dessa vertente, pelo menos não em termos práticos e resultados objetivos. O crítico literário indiano R. Shashidhar classificou a perspectiva de Williams em Cultura e Sociedade como "organicismo sistêmico". O princípio com o qual o galês trabalhou no livro conteria: um elemento histórico (o contexto, a "fonte"), um elemento linguístico (o conteúdo - as declarações pessoais dos pensadores subsequentes, incluindo as do próprio Williams - distingue significados ao praticá-los de maneiras relevantes no presente) e, como figura central, um elemento existencial, o elemento da consciência, que "responde" ao contexto imediato da experiência através de palavras-chave herdadas (SHASHIDHAR, 1997, p. 42).

Esse organicismo sistêmico viria, então, carregado de carências de Williams no campo da tradição radical, dentre eles a falta de entendimento dos conceitos sociedade civil, luta de classes e conflito de classes. Williams teria tomado a linguagem como um tipo de index orgânico de uma entidade igualmente orgânica chamada "sociedade". Não haveria dúvidas de que o discurso que ele traçou em Cultura e Sociedade registrou as mudanças ocorridas, mas a questão mais importante seria: do ponto de vista de quem? (SHASHIDHAR, 1997, p. 42-43).

Nesse sentido, apesar do título, não haveria muito de sociedade no livro, a não ser quando aparece como uma preocupação ético-cultural dos autores discutidos, fenomenologicamente processada por Williams ${ }^{6}$. Esse tipo de leitura também está em E. P. Thompson, que enfatizou que Cultura e Sociedade poderia, por vezes, ser visto como uma "procissão de vozes desencarnadas" (THOMPSON, 1961, p. 24).

Em concordância com Stuart Hall, acredito que Williams foi certeiro ao identificar o potencial da "crítica prática" de Cambridge e o modo como essa metodologia

6. Cf. Ibidem, p. 48 . 
O "materialismo cultural" de Raymond Williams a partir da história...

buscava enfatizar a complexidade envolta na análise cultural em comparação com a crítica cultural marxista britânica do período. Williams também soube reconhecer a força que a tradição da crítica de Cambridge exercia sobre quem foi treinado em seus moldes (HALL, 1989, p. 3-7), e isso foi justamente o que aconteceu em Cultura e Sociedade. Ainda segundo Hall, a obra teria sido profundamente marcada pela tradição a que queria se contrapor, e isso fica ainda mais evidente em seu método, que conseguiu apontar o "idioma da tradição", mas não conseguiu ir além do discurso empírico-moral. A questão, no caso inglês, não se localizaria somente na esfera cultural, mas sim no modo pelo qual um conjunto particular de valores sociais e políticos se sedimentou em uma inflexão habitual de linguagem e pensamento. Esse livro não conseguiu pontuar isso, e nem conseguiria, porque ele teria permanecido, de certa maneira, preso metodologicamente dentro da análise discursiva (HALL, 1989, p. 59).

Quais são as causas dessa armadilha metodológica? Minha aposta se encontra justamente em seu fraco tratamento dos diferentes contextos históricos e políticos. Hall postulou que um dos maiores problemas do livro se encontraria na falta de elementos não-britânicos, especialmente no que diz respeito ao impacto da Revolução Francesa. Outra questão apontada por ele se refere a uma possível falta de contraposição das ideias dos autores analisados com outras correntes de pensamento do período (tais como: política econômica, individualismo político, liberalismo e empirismo), pois assim seria possível refletir a respeito dos termos de sua formação e de qual seria o caráter excepcional dessa tradição intelectual, assim localizando-a socialmente e historicamente (HALL, 1989, p. 60).

A partir dessas ponderações é possível pensar em como a concepção de história de Williams opera e no modo como ela foi posta em prática. Minhas reflexões acerca disso ainda são bastante preliminares; no entanto, tendo a inferir que o posicionamento de Williams possui muitas características similares ao de Arthur Lovejoy e sua história das ideias. O filósofo e historiador germano-americano fez uma série de palestras durante o ano de 1933, depois foram publicadas na coletânea chamada A grande cadeia do ser (1936). De modo geral, Lovejoy apresentou uma concepção e metodologia para o estudo do pensamento, em que ideias são "fatores dinâmicos" e sua proposta consistia em analisar esses elementos e identificar "as unidades dinâmicas persistentes ou recorrentes", o que ele chamou de "ideias-unidade" (Unit Ideas) (LOVEJOY, 1936, p. 5-7).

Outros autores já apontaram para elementos da história das ideias contidos no trabalho de Williams, tais como Beatriz Sarlo (WWE/2/1/7/3/4), por exemplo, 
que o denominou especificamente de "historiador das ideias". No entanto, pergunto-me qual o impacto disso na análise dos autores selecionados e, por consequência, na noção de cultura enquanto "conceito-ideia' em Cultura e Sociedade. Graham Martin defendeu que é óbvio que a análise do livro é uma história das ideias, no entanto, seria muito mais do que isso, pois a abordagem por detrás de tal prática sugeriria, por vezes, uma omissão deliberada sobre como essas surgem dentro de um contexto histórico específico e, após isso, é realizado um "resgate" pelos que foram influenciados posteriormente. Em Cultura e Sociedade, então, as ideias se tornariam ativas dentro da observação social do pensador, esclarecendo ou confundindo, afiando ou cegando a resposta de seus "sentimentos", revelando seu "tom" e "idioma". Isso só seria possível pelo treino do crítico literário e o uso específico dessa metodologia (WWE/2/1/9/2/6, p. 71).

É curioso como o que é exaltado por Martin é justamente o ponto criticado por Hall mencionado anteriormente, o foco no discurso (HALL, 1989, p. 59). Seria possível articular a posição da análise histórica e política na obra de Williams com certa abordagem da história das ideias que privilegia tal tratamento das ideias e do pensamento?

Logo após a publicação do livro em 1958, Victor Kiernan escreveu uma resenha bastante crítica dele. Não creio que ele tinha as mesmas preocupações que eu, mas seu posicionamento frente à questão do uso ineficiente do contexto histórico por Williams traz elementos importantes para a discussão. Segundo o autor, o primeiro requisito para qualquer estudo de história cultural residiria na firme estrutura de fatos históricos, econômicos, sociais e políticos. Dito isso, Kiernan enfatizou como o galês teria posicionado como central o processo da Revolução Industrial; no entanto, não teria ficado claro o que ele entendia por isso (WWE/2/1/9/2/6, p. 75-76). Tais abordagens, silêncios e pressuposições afetariam a possibilidade de relacionar analiticamente pensamento e sociedade. Kiernan enfatizou que seria necessário, então:

Uma investigação mais avançada na história da cultura combinada com a história da classe "cultural". A relativa autonomia da arte e da filosofia da compulsão econômica de sua época é o mesmo problema, só que em outro plano, da relativa autonomia da intelligentsia dentro da estrutura de poder da sociedade de classes (WWE/2/1/9/2/6, p. 83).

De acordo com Hall, discutir o que Cultura e Sociedade deixou de lado virou quase um jogo intelectual (HALL, 1989, p. 60). Acredito que isso se deve ao fato de o livro 
conseguir incorporar tão bem as complexidades e ambivalências do pensamento de seu autor, bem como da esquerda do pós-guerra britânico de modo geral. Vale a pena mencionar que quase vinte anos após sua publicação, o próprio Williams refletiu sobre como, naquela época, não tinha em mãos o conhecimento histórico necessário para embasar suas análises; disse ainda que se sentia muito distante da obra e que parecia que outra pessoa a escreveu (WILLIAMS, 1979, p. 107-108).

Tais considerações, em conjunto com a própria autocrítica de Williams, são importantes para refletir sobre seu trajeto intelectual posterior e como Williams foi ao encontro de proposições centrais do marxismo ocidental, seja através do contato com obras fundamentais dessa tradição, ou da guinada teórica da esquerda da Europa continental. É fato que seu trabalho da década de 1970, mais especificamente Marxismo e Literatura, apresenta uma combinação de sofisticação teórica e amadurecimento intelectual sem precedentes, uma voz autoral marcante que agora clama seu lugar dentro da tradição teórica do marxismo. A pesquisa aqui delineada tenta apontar elementos dessa mudança no campo e certas estruturas de sentimento presentes e "cristalizadas" nesse livro, que marcou lugar na história intelectual ocidental e necessita uma investigação dedicada.

A pesquisa está sendo marcada pela descoberta e redescoberta das ideias de Williams ao colocá-las à luz de seu contexto. Ao mesclar tradição e inovação, o autor não só trouxe novos objetos e novos temas às ciências humanas; trouxe também novas ferramentas, além do resgate, da revisão e da atualização de conceitos e categorias cruciais para a análise da sociedade capitalista contemporânea ocidental, na qual a cultura tem papel de destaque. Seu legado se estende para além das barreiras disciplinares, e no que diz respeito especificamente à história, o materialismo cultural tem muito a dizer, tanto como método de investigação quanto como discussão epistemológica.

\section{REFERÊNCIAS}

\section{Richard Burton Archives - RBA}

WWE/2/1/9/2/6 - Resenhas de Victor Kiernan (publicada no The New Reasoner, no verão de 1958) e resenha de Graham Martin (publicada no Universities and Left Review, no verão de 1958). WWE/2/1/7/3/4 - Entrevista por Beatriz Sarlo, de título: "Raymond Williams y Richard Hoggart: sobre cultura y sociedade", Revista Punto de Vista, ano 2, número 6, julho de 1979. 


\section{OBRAS}

BOURDIEU, Pierre. Campo intelectual e projeto criador. In: POUILLON, Jean (Org.). Problemas do estruturalismo. Rio de Janeiro: Zahar, 1968, p. 105-145. . Algumas propriedades dos campos. Questões de sociologia. Lisboa: Fim de século, 2006. . Razões Práticas: sobre a teoria da ação. Campinas: Papirus, 1996. . As regras da Arte: gênese e estrutura do campo literário. São Paulo: Cia das Letras, 2002. CEVASCO, Maria E. Para ler Raymond Williams. São Paulo: Paz e Terra, 2001.

EAGLETON, Terry. Criticism and ideology: a study in Marxist Literary Theory. London: Verso, 1976. . Resources for a journey of Hope: significance of Raymond Williams. New Left Review, n. 168, mar./abr. 1988, p. 3-11.

ESCOSTEGUY, Ana C. D. Uma introdução aos Estudos Culturais. Revista Famecos, n. 9, dez. 1998, p. 87-97.

GARNHAM, Nicholas; WILLIAMS, Raymond. Pierre Bourdieu and the sociology of culture: an introduction. Media, Culture and Society, v. 2, n. 3, 1980, p. 209-223.

HALL, Stuart. Politics and Letters. In: EAGLETON, T. (Org.) Raymond Williams: critical perspectives. Cambridge: Polity Press, 1989. p. 54-66.

LOVEJOY, Arthur. The Great Chain of Being: a study of the history of an idea. Cambridge (EUA): Harvard Univ. Press, 1936.

MEYERS, Jorge. Músicas distantes. Algumas notas sobre a História Intelectual hoje: horizontes velhos e novos, perspectivas que se abrem. In: NORONHA DE SÁ, M. E. (Org.). História Intelectual Latino-Americana: Itinerários, debates e pesquisas. Rio de Janeiro: Ed. PUC Rio, 2016. p. 23-55.

MILNER, Andrew. Re-imagining Cultural Studies: the promise of Cultural Materialism. London: Sage, 2002.

MULHERN, Francis. The moment of Scrutiny. London: Verso, 1979;

PASSIANI, Enio. Afinidades seletivas: uma comparação entre as sociologias da literatura de P. Bourdieu e R. Williams. Estudos de Sociologia, v. 14, n. 27, 2009, p. 285-299.

SHASHIDHAR, R. Culture and Society: an introduction to Raymond Williams. Social Scientist, v. 25, n. 5/6, 1997, p. 33-53.

THOMPSON, E. P. The Long Revolution - Part I. New Left Review, n. 9, maio-jun. 1961, p. 24-33. WILLIAMS, Raymond. The Bloomsbury Fraction. In: . Culture and materialism. London:

Verso Books, 1980, p. 148-169. .Culture and Society: 1780-1950. New York: Anchor Books/Columbia University Press, 1963. . The Long Revolution. Harmondsworth: Penguin Books, 1961, p. 19-144. . Marxism and literature. London: Oxford University Press, 1984. . Notes on Marxism in Britain since 1945. New Left Review, I/100, nov./dez. 1976, p. 81-94. . Politics and Letters: interviews with New Left Review. London: Verso, 1979. 
O "materialismo cultural" de Raymond Williams a partir da história...

SOBRE A AUTORA

Carla Baute é graduada em História (Universidade Federal de São Paulo) e atualmente é mestranda em História (Universidade Estadual de Campinas). Tem experiência na área de História Intelectual, com pesquisa nos seguintes temas: Raymond Williams, Materialismo Cultural, New Left, Estudos Culturais e Marxismo Ocidental. Financiada pela Fundação de Amparo à Pesquisa do Estado de São Paulo (FAPESP).

E-mail: carlabaute@gmail.com.

Recebido em o1 de julho de 2019 e aprovado em 23 de setembro de 2019. 